\title{
Exact reduced dynamics for a qubit in a precessing magnetic field and in the contact with a heat-bath
}

\author{
Bartłomiej Gardas* \\ Institute of Theoretical and Applied Informatics, \\ Polish Academy of Sciences, Battycka 5, 44-100 Gliwice, Poland
}

(Dated: 26 May 2010)

\begin{abstract}
The two-level quantum system (qubit) in a precessing magnetic field and in contact with a heat bath is investigated. The exact reduced dynamics for the qubit in question is obtained. We apply the approach based on the block operator matrices theory, in contrast with the standard methods provided by the theory of the open quantum systems. We also present the solution of the Riccati operator equation associated with the Hamiltonian of the system. Next, we study the adiabatic approximation for the system under consideration using quantum fidelity as a way to measure validity of the adiabatic theory. We find that in the weak coupling domain the standard condition that ensures adiabatic behavior of the spin in the magnetic field also guarantees its adiabatic evolution in the open system variant of this model. Therefore, we provide the explicit example of the open quantum system that satisfies the adiabatic approximation firstly formulated for the closed quantum systems.
\end{abstract}

PACS numbers: 03.65.Yz, 03.67.-a

Keywords: Riccati equation; Exact reduced dynamics; rotating magnetic field; adiabatic approximation

\section{INTRODUCTION}

The most established and useful time-dependent twolevel quantum system, is perhaps, the one that describes spin half particle (qubit $Q$ ) in a precessing magnetic field [1]. The Hamiltonian of this system in its basic variant is usually written in the following form

$$
H_{Q}(t)=-\gamma \mathbf{S} \cdot \mathbf{B}(t), \quad \mathbf{S}=\boldsymbol{\sigma} / 2,
$$

where $\gamma$ is a constant, called the gyromagnetic ratio (the specific value of $\gamma$ is irrelevant in context of our discussion $), \boldsymbol{\sigma} \equiv\left(\sigma^{x}, \sigma^{y}, \sigma^{z}\right)$ and $\sigma^{i},(i=x, y, z)$ are the standard Pauli matrices. The magnetic field $\mathbf{B}(t)$ is assumed to have the form

$$
\mathbf{B}(t)=B_{1} \cos (\omega t) \hat{e}_{x}+B_{1} \sin (\omega t) \hat{e}_{y}+B_{0} \hat{e}_{z},
$$

where $\hat{e}_{x}, \hat{e}_{y}, \hat{e}_{z}$ are the unit vectors along $x, y$ and $z$ axis, respectively. $B_{0}, B_{1}$ define the amplitudes of the magnetic field and $\omega$ is the frequency of the rotation. Using the equations above one can also rewrite the Hamiltonian (1) in a more readable form:

$$
H_{Q}(t, \beta)=\beta \sigma_{3}+\alpha\left(\sigma_{2} \sin (\omega t)+\sigma_{1} \cos (\omega t)\right),
$$

where for the sake of convenience the abbreviations $\alpha=$ $\frac{1}{2} \omega_{1} \equiv-\frac{1}{2} \gamma B_{1}$ and $\beta=\frac{1}{2} \omega_{0} \equiv-\frac{1}{2} \gamma B_{0}$ were introduced.

In the case of no coupling with the external environment (heat-bath) the exact form of the evolution

\footnotetext{
* bartek.gardas@gmail.com
}

operator $U_{Q}(t)$ and hence the density matrix $\rho_{Q}(t)=$ $U_{Q}(t) \rho_{Q} U_{Q}(t)^{\dagger}$ for the model $(3)$, can be derived in an elegant and simple manner (see e.g. [1, 2]). This problem is so common that it can be found in almost every modern textbook on quantum mechanics. However, if the aforementioned coupling is present, the exact form of the density matrix has not yet been derived.

If one allows the system to interact (not necessarily to exchange the energy) with the environment then it becomes so called open quantum system [3]. Its time evolution is not unitary any more because of the decoherence process [4, 5]. Nevertheless, it may always be described by trace preserving (TP) and completely positive (CP) quantum operation (or quantum channel, see e.g. [6]) $T_{t}: \rho_{Q} \rightarrow \rho_{Q}(t), \rho_{Q}(0) \equiv \rho_{Q}$. Unfortunately, finding its exact form is almost impossible in most cases, especially for the systems described by the time-dependent Hamiltonian.

Naturally the question arises: how difficult this problem actually is? Recently, the efforts to answer this question were made $[7,8]$. It was shown that from the mathematical perspective this task is at least as complicated as the problem of resolving the Riccati algebraic equation associated with the Hamiltonian defining the model in question. Moreover, from the block operator matrices theory point of view obtaining the exact reduced dynamics for any system where decoherence is present (beyond dephasing phenomenon) $[9,10]$ is as complex as resolving this equation. Of course, the complexity of the analysis strongly depends on the particular choice of the environment responsible for the decoherence process.

First, we show that all the difficulties mentioned above, including the problem of resolving the Riccati equation, can be overcome for the so-called spin environment. Therefore, we provide the exact reduced dynamics for the open system version of the model (1) in the case when 
the system is immersed within the spin-bath. Next, as an application, we investigate the adiabatic approximation for the open system constructed in this way. Moreover, we show that in the weak coupling domain the condition ensuring the adiabatic evolution of the system (1) also leads to the adiabatic behavior of its generalization.

The layout of this paper is as follows. In Sec. (II) we briefly review the concept of the block operator matrix and the procedure allowing one to diagonalize it. In Sec. (II A) we introduce the Riccati operator equation and discuss its connection with the Hamiltonian specifying the model. In Sec. (III) the definition of the model is given and the exact reduced dynamics is derived. We also indicate possible applications of the model. In Sec. (V) we investigate the adiabatic approximation. Finally, Sec. (VI) contains the final remarks and the summary of the paper.

\section{BLOCK OPERATOR MATRICES APPROACH}

\section{A. Partial trace and reduced dynamics}

Before we can go any further and present the main result of this paper let us first discuss the procedure allowing one to obtain the density matrix $\rho_{Q}(t)$ for a twodimensional system (qubit) using the block operator matrices approach.

Let $H_{Q E}$ be the Hamiltonian of the closed (qubit and environment, $Q+E$ ) system. We will assume its following form

$$
H_{Q E}=H_{Q} \otimes \mathbb{I}_{E}+\mathbb{I}_{Q} \otimes H_{E}+H_{\text {int }},
$$

where $H_{Q}, H_{E}$ are the Hamiltonian of the qubit and the environment respectively, while $H_{\text {int }}$ represents the interaction between the systems. Hamiltonian (4) acts on $\mathcal{H}_{Q E}=\mathcal{H}_{Q} \otimes \mathcal{H}_{E}$ space, where $\mathcal{H}_{Q}$ and $\mathcal{H}_{E}$ are the Hilbert spaces for the qubit and the environment, respectively. The state $\rho_{Q}(t)$ of the qubit at any given time $t$ can be computed using the following formula (for more details see e.g. [11] )

$$
\begin{aligned}
\rho_{Q}(t) & =\operatorname{Tr}_{E}\left(U_{t} \rho_{Q} \otimes \rho_{E} U_{t}^{\dagger}\right) \\
& \equiv T_{t}\left(\rho_{Q}\right)
\end{aligned}
$$

where $U_{t}=\exp \left(-i H_{Q E} t\right)$ is the evolution operator of the total $Q+E$ system (we work with units $\hbar=1$ ). By $\operatorname{Tr}_{E}(\cdot)$ we denoted the partial trace. In the literature the quantity $\rho_{Q}(t)$ is commonly called the reduced dynamics. In this paper we will be also referring to it as the solution of a given model. In Eq. (5) the assumption that $\rho_{Q E}=$ $\rho_{Q} \otimes \rho_{E}$ was made, i.e., no correlation between $Q$ and $E$ are present initially (see e.g., $[12,13]$ and references therein).
Since $\mathcal{H}_{Q}=\mathbb{C}^{2}$ and the isomorphism $\mathbb{C}^{2} \otimes \mathcal{H}_{E}=$ $\mathcal{H}_{E} \oplus \mathcal{H}_{E}$ holds, one can think of the operator $H_{Q E}$ (and of any given operator $A$ acting on $\mathcal{H}_{Q} \otimes \mathcal{H}_{E}$ ) as a $2 \times 2$ block operator matrix [14]. Let us assume that $A$ acts on $\mathcal{H}_{E} \oplus \mathcal{H}_{E}$. Thus, we can write $A=\left[A_{i j}\right]$, where operators $A_{i j},(i, j=1,2)$ act on $\mathcal{H}_{E}$. In this block operator matrices nomenclature the procedure of calculating the partial trace $\operatorname{Tr}_{E}(\cdot)$ can be written as

$$
\operatorname{Tr}_{E}(A)=\left(\begin{array}{ll}
\operatorname{Tr} A_{11} & \operatorname{Tr} A_{12} \\
\operatorname{Tr} A_{21} & \operatorname{Tr} A_{22}
\end{array}\right)
$$

where $\operatorname{Tr}(\cdot)$ denotes the trace on $\mathcal{H}_{Q}$. Eq. (6) introduces the concept of the partial trace in a very simple and, what is the most important, intuitive way. It is also possible to define the partial trace locally, i.e., $\operatorname{Tr}_{E}(X \otimes Y)=X \operatorname{Tr}(Y)$, where as mentioned before $\operatorname{Tr}(\cdot)$ stands for the trace operation on the space on which the operator $Y$ acts on. From Eq. (6) one can learn that the partial trace is the operation which transforms operator matrices into the "ordinary" matrices. Moreover, the partial trace is a linear operation going from $B\left(\mathcal{H}_{Q E}\right)$ space to the $B\left(\mathbb{C}^{2}\right)$ space. Furthermore, from the block operator matrices perspective one can easily understand why dealing with the open systems is much more complex (from the mathematical viewpoint) then dealing with the closed systems. The reason is that the procedure allowing one to calculate the reduced dynamics is not as straightforward as Eq. (6) might indicate. Indeed, to compute the partial trace (5) one needs to write the evolution operator $U_{t}$ in a $2 \times 2$ block matrix form. The last operation requires diagonalization of the block operator matrix $H_{Q E}$, which is much more complicated than the diagonalization of the standard $2 \times 2$ matrix. It leads to the problem with solving the Riccati algebraic equation analyzed below.

\section{B. Riccati equation and block diagonalization}

With every Hermitian block operator matrix of the form

$$
R=\left[\begin{array}{cc}
A & B \\
B^{\dagger} & C
\end{array}\right]
$$

where $A, B, C$ are the Hermitian operators acting on $\mathcal{H}_{E}$, one can associate the following Riccati equation [15]

$$
X B X+X A-C X-B^{\dagger}=0,
$$

where $X \in B\left(\mathcal{H}_{E}\right)$ is the operator to be determined.

If the solution $X$ of the Eq. (8) exists, then it may be used to diagonalize operator matrix (7). The procedure that allows us to do so is as follows (for details see [8] and references therein) 


$$
U_{X}^{-1} R U_{X}=\left[\begin{array}{cc}
A+B X & 0_{E} \\
0_{E} & C-(X B)^{\dagger}
\end{array}\right],
$$

where the similarity matrix $U_{X}$ is given by

$$
U_{X}=\left[\begin{array}{cc}
\mathbb{I}_{E} & -X^{\dagger} \\
X & \mathbb{I}_{E}
\end{array}\right]
$$

We wish to emphasize that in most cases a general method of finding the solution for the Riccati equation does not exist. Therefore, resolving the Eq. (8) with respect to $X$ is a very difficult task. Moreover, even if the solution is known, there is still a problem of computing the inversed operator matrix $\left(U_{X}\right)^{-1}$. Formally, if the operator $X$ is normal, i.e., $\left[X, X^{\dagger}\right]=0$, then this matrix is given by the formula

$$
U_{X}^{-1}=\left(\mathbb{I}_{E}+X X^{\dagger}\right)^{-1}\left[\begin{array}{cc}
\mathbb{I}_{E} & X^{\dagger} \\
-X & \mathbb{I}_{E}
\end{array}\right]
$$

Otherwise, the form of the inverse operator $U_{X}^{-1}$ becomes more complicated. Fortunately, the Eq. (11) is sufficient for our analysis. Notice that it may be difficult to handle computationally the operator such as $\left(\mathbb{I}_{E}+X X^{\dagger}\right)^{-1}$. In general the operator $X$ does not need to be Hermitian or unitary.

Perhaps those are the reasons why the approach to open systems based on the operator matrices theory has not attracted too much attention. In our opinion, however, the operator matrix perspective can offers a better understanding of the open quantum systems and might give some new results in this area.

\section{EXACT REDUCED DYNAMICS}

\section{A. Model}

We consider the model defined by the following timedependent Hamiltonian

$$
H_{Q E}(t, \beta)=H_{Q}(t, \beta) \otimes \mathbb{I}_{E}+\mathbb{I}_{Q} \otimes H_{E}+H_{\text {int }},
$$

where $H_{Q}(t, \beta)$ is given by Eq. (3) and it represents a qubit in the magnetic field (2). The environment is composed of $N$ independent and non-interacting spin $1 / 2$ particles. The Hamiltonian $H_{E}$ of the bath is assumed to be of the form

$$
H_{E}=\sum_{n=1}^{N} \omega_{n} \sigma_{n}^{z}
$$

where $\omega_{n}$ and $\sigma_{n}^{z},(n=1, \ldots, N)$ are the frequencies and the Pauli matrices for $n$th qubit, respectively. The
Hilbert space $\mathcal{H}_{E}$ on which the Hamiltonian (13) acts is given by $N$-fold tensor product of $\mathbb{C}^{2}$ spaces, i.e., $\mathcal{H}=\bigotimes_{n=1}^{N} \mathbb{C}^{2}$. Therefore for any $n \leq N$ the operator $\sigma_{n}^{z}$ is understood as $\sigma_{n}^{z}=I_{2} \otimes \ldots \otimes \sigma^{z} \otimes \ldots \otimes I_{2}$, where $\sigma^{z}$ is the standard Pauli matrix acting on $\mathbb{C}^{2}$ and $I_{2}$ is the $2 \times 2$ identity matrix on that space. In our model the coupling of a qubit with the environment is governed by the Ising-typ Hamiltonian [16, 17], namely

$$
H_{i n t}=\sigma^{z} \otimes \sum_{n=1}^{N} g_{n} \sigma_{n}^{z}
$$

where $g_{n}$ are the coupling constants. We assume that the bath is initially in the Gibbs thermal state at a temperature $T$, i.e.,

$$
\rho_{E}=Z^{-1} \exp \left(-H_{E} / k T\right),
$$

where $Z=\operatorname{Tr}\left(\exp \left(-H_{E} / k T\right)\right)$ and $k$ is the Boltzmann constant.

The model described by the Hamiltonian (12) with $H_{E}$ and $V$ specified by the Eqs. (13)-(14) in the case of the $\alpha=0$ (i.e., dephasing and static magnetic field case) was investigated both in the context of the approximation methods in the open quantum systems theory [16] and the capacities of the quantum channels [17]. Thus, for the detailed discussion and possible applications we refer the reader therein.

The quantum system discussed here can by thought of as the generalization of the model introduced in the mentioned papers to the case when the energy is exchanged between the systems and the magnetic field depends on time. It is worth mentioning physical problems that make use of this model. First of all, the Hamiltonian (12) may pose a useful prototype describing any spin "resonance" phenomenon, like for instance Nuclear Magnetic Resonance (NMR) $[18,19]$. In such picture the spin $1 / 2$ particle is the open system, which evolution in time we wish to describe. The spin-bath models the influence of the other spins on the open system in question. Finally, the rotating magnetic field is used to induce the resonance and to control the system.

The quantum devices of the future, like for instance quantum computers [20] will be probably built from the components that are composed of a large amount of the qubits (spins, in particular) e.g., quantum memory [21], quantum register [22], etc. The model we are investigating may serves as a simple prototype allowing one to trace the evolution in time of a single qubit of the register or memory. The magnetic field may be then applied to program the device. Those are just a few potential applications of the simple theoretical, time-dependent spin-spins model (12). 


\section{B. Exact solution}

To derive an exact reduced dynamics of the system governed by the Hamiltonian (12) we first simplify this problem to a time-independent one. In order to accomplish this we use recently proved theorem that says if (for more details see (A) and also [8])

i) the total system is defined by the Hamiltonian (12),

ii) the interaction term $H_{\text {int }}$ between the systems takes the form $f\left(\sigma^{z}\right) \otimes V$, where $f$ is an analytical function of $\sigma^{z}$ and $V$ is a Hermitian operator,

iii) $\rho_{t}(\beta)$ represents the solution of the system defined by the Hamiltonian $H_{Q E}(0, \beta)$ (note that Hamiltonian $H_{Q E}$ depends on $\beta$, hence so is $\rho_{t}$ ),

then the reduced dynamics $\eta_{t}$ for the model governed by the Hamiltonian (12) can be obtained using the following simple formula

$$
\eta_{t}=V_{t} \rho_{t}\left(\beta_{e f f}\right) V_{t}^{\dagger}, \quad \beta_{e f f}:=\beta-\omega / 2,
$$

where the unitary matrix $V_{t}$ is given by (diag-diagonal)

$$
V_{t}=\operatorname{diag}\left(e^{-i \omega t / 2}, e^{i \omega t / 2}\right)
$$

Equality (16) states that having the reduced dynamics $\rho_{t}$ one may easily obtain the solution we are interested in simply by introducing the effective parameter $\beta_{\text {eff }}$, then replacing $\beta$ by $\beta_{\text {eff }}$ and finally performing the unitary transformation (17).

The interaction term defined in the Eq. (14) satisfies the requirement of the theorem above, thus one should restrict the analysis to the Hamiltonian $H_{Q E}(\beta) \equiv$ $H_{Q E}(0, \beta)$. The later can be easily rewritten as a $2 \times 2$ block operator matrix, namely

$$
H_{Q E}(\beta)=\left[\begin{array}{cc}
H_{+}(\beta) & \alpha \mathbb{I}_{E} \\
\alpha \mathbb{I}_{E} & H_{-}(\beta)
\end{array}\right],
$$

where

$$
H_{ \pm}(\beta):=\sum_{n=1}^{N}\left(\omega_{n}^{ \pm} \sigma_{n}^{z} \pm \bar{\beta} \mathbb{I}_{E}\right), \quad \bar{\beta}:=\beta / N
$$

and $\omega_{n}^{ \pm}:=\omega_{n} \pm g_{n}$, for each $n \leq N$. Henceforward, the explicit dependence of any quantity of parameter $\beta$ will be omitted until the solution $\rho_{Q}(t)$ is obtained.

The evolution operator for the total system generated by the Hamiltonian (18) reads

$$
\begin{aligned}
U_{t} & =\exp \left(-i H_{Q E} t\right) \\
& =U_{X} \exp \left(-i H_{d}\right) U_{X}^{-1},
\end{aligned}
$$

where $U_{X}$ is given by the Eq. (10) and $X$ is the solution of the Riccati Eq. (8), which in the present case takes the form

$$
\alpha X^{2}+X H_{+}-H_{-} X-\alpha \mathbb{I}_{E}=0 .
$$

The quantity $H_{d}$ represents a diagonal form of the operator matrix $H_{Q E}$. According to the Eq. (9) it reads

$$
H_{d}=\left[\begin{array}{cc}
H_{+}+\alpha X & 0_{E} \\
0_{E} & H_{-}-\alpha X^{\dagger}
\end{array}\right]
$$

Note that for $\alpha=0$ the solution (possibly not the only one) of the Eq. (21) is given by $X_{0}=0_{E}$. It is obvious, since in that case $H_{Q E}$ is already in the diagonal form, i.e., $H_{Q E}=H_{d}=\operatorname{diag}\left(H_{+}, H_{-}\right)$. In order to obtain the solution for $\alpha \neq 0$ more subtle investigation, provided in the next subsection, is needed.

\section{Solution of the Riccati equation for the $\alpha \neq 0$ case}

First, one can observe that $\left[H_{-}, H_{+}\right]=0$. Since the solution $X$ of the Eq. (21) is a function of the operators $H_{ \pm}$, then it also must commute with those operators. The Riccati equation in question can be simplified to the more compact form, namely

$$
\alpha X^{2}+2 V X-\alpha \mathbb{I}_{E}=0, \quad \alpha \neq 0,
$$

where we introduced

$$
V=\sum_{n=1}^{N}\left(g_{n} \sigma_{n}^{z}+\bar{\beta} \mathbb{I}_{E}\right) .
$$

Let us assume that the eigenvalue problem for the operator $V$ can be (easily) resolved. If this is the case we can write the solution $X$ of the Eq. (23) in a manageable form using the spectral theorem for the Hermitian operators. By doing so we obtain that $X=f(V)$, where

$$
f(\lambda)=\frac{\sqrt{\lambda^{2}+\alpha^{2}}-\lambda}{\alpha}, \quad \lambda \in \sigma(V) .
$$

We can also represent the solution $f(V)$ in an equivalent way, i.e.

$$
f(V)=\sum_{\lambda \in \sigma(V)} f(\lambda)|\lambda\rangle\langle\lambda| .
$$

Note that $f=f^{*}$, i.e., $f$ is a real function for any value of the parameters $\alpha$ and $\beta$. Here, by $\sigma(V)$ we denoted the spectrum of $V$. We want to emphasize that the representation (25) or (26) of the solution $X$ of the Eq. (23) 
is useful computationally if the eigenvalues of $V$ can be computed. We show below that this is the case. Note also that $X=X^{\dagger}$ iff $V=V^{\dagger}$.

Let $i=i_{1} i_{2} \ldots i_{N}$ be a binary $\left(i_{n}=0,1\right.$, for $\left.n \leq N\right)$ expansion of an integer number $i \in\left[0,2^{N}-1\right]$. Clearly, the set of all eigenstates $|i\rangle=\left|i_{1}\right\rangle \otimes\left|i_{2}\right\rangle \otimes \ldots \otimes\left|i_{N}\right\rangle$ forms an orthonormal basis in $\mathcal{H}_{E}$. Moreover, because of the following equality $\sigma^{z}\left|i_{n}\right\rangle=(-1)^{i_{n}}\left|i_{n}\right\rangle$ we have

$$
\begin{aligned}
V|i\rangle & =\sum_{n=1}^{N}\left(g_{n}(-1)^{i_{n}}+\bar{\beta}\right)|i\rangle \\
& \equiv E_{i}|i\rangle, \quad 0 \leq i \leq 2^{N}-1 .
\end{aligned}
$$

Thus, the eigenvalue problem for $V$ has been solved. Therefore, the solution of the Riccati Eq. (23) $X$ reads

$$
X=\sum_{i=0}^{2^{N}-1} f\left(E_{i}\right)|i\rangle\langle i| .
$$

At this point two remarks should by made. The first one is that the operator (28) depends on the parameter $\alpha$, i.e., $X=X(\alpha)$ and $X(\alpha) \rightarrow X_{0}$ as $\alpha$ goes to 0 . To see that this statement holds we rewrite the Eq. (25) in the form

$$
f(\lambda)=\frac{1}{\sqrt{\left(\frac{\lambda}{\alpha}\right)^{2}+1}+\frac{\lambda}{\alpha}}, \quad \lambda \in \sigma(V) .
$$

Clearly, $f(\lambda) \rightarrow 0$ as $\alpha \rightarrow 0$, unless $\lambda=0$. The later means that the solution $X$ of the Riccati Eq. (23) is a continuous function of the parameter $\alpha$, including the $\alpha=0$ value. From the physical point of view it means that one can control energy exchange between the systems in a continuous way. Note that if $\alpha=0$ then the energy transfer is absent. This scenario can be accomplished by taking limits $\alpha \rightarrow 0$ with the final solution we are about to give. Note also that since for every $\alpha \neq 0$ and each $\lambda \in \sigma(V)$ the eigenvalues $f(\lambda)$ of the operator $X$ we constructed are positive. This solution is a positively defined operator on $\mathcal{H}_{E}$.

The second remark is that there exists, at least one more operator function that satisfies the Eq. (23), namely the one specified by the following formula: $f_{2}(\lambda)=$ $\left(-\sqrt{\lambda^{2}+\alpha^{2}}-\lambda\right) / \alpha$ for $\lambda \in \sigma(V)$. This solution represents negatively defined operator. The choice between the different solution of the Eq. (23) is not arbitrary and it has significant influence on the further analysis. Indeed, if one decides to choose the second solution: $f_{2}(\lambda)$ then one may meet a serious difficulties during the examination of the dephasing phenomena, since $f_{2}(\lambda) \rightarrow-\infty$ as $\alpha \rightarrow 0$.

At the end of this subsection let us note that one can resolve the eigenvalue problem for $H_{ \pm}$as easily as for the operator $V$. Indeed, we may readily verify that

$$
\begin{aligned}
H_{ \pm}|i\rangle & =\sum_{n=1}^{N}\left(\omega_{n}^{ \pm}(-1)^{i_{n}} \pm \bar{\beta}\right)|i\rangle \\
& \equiv E_{i}^{ \pm}|i\rangle, \quad 0 \leq i \leq 2^{N}-1 .
\end{aligned}
$$

Observe also that $E_{i}^{+}-E_{i}^{-}=2 E_{i}$, for $0 \leq i \leq 2^{N}-1$, as one may expected. In the $|i\rangle$ basis the density matrix $\rho_{E}$ can be expanded as

$$
\rho_{E}=\sum_{i=0}^{2^{N}-1}\left(e^{-\Omega_{i} / k T} / Z\right)|i\rangle\langle i|,
$$

where $\Omega_{i}=\sum_{n} \omega_{n}(-1)^{i_{n}}$ are the eigenvalues of $H_{E}$ and $Z=\sum_{i} \exp \left(-\Omega_{i} / k T\right)$.

\section{Total system evolution}

We are now in a position to give the explicit and manageable form of the evolution operator of the total system $Q+E$. First, let us note that the similarity operator matrix $U_{X}$ can by written as

$$
U_{X}=\sum_{i=0}^{2^{N}-1} U_{i} \otimes|i\rangle\langle i|,
$$

where matrices $U_{i}$ are given by

$$
U_{i}=\left(\begin{array}{cc}
1 & -f\left(E_{i}\right) \\
f\left(E_{i}\right) & 1
\end{array}\right) .
$$

This follows immediately from the Eqs. (10) and (28). We also used the resolution of the identity $\mathbb{I}_{E}$ in the $|i\rangle$ basis. From the expansion (32) one can readily see that the inversed operator $\left(U_{X}\right)^{-1}$ takes the form

$$
\left(U_{X}\right)^{-1}=\sum_{i=0}^{2^{N}-1}\left(U_{i}\right)^{-1} \otimes|i\rangle\langle i|,
$$

where $\left(U_{i}\right)^{-1}$ stands for the inverse of the matrix $U_{i}$. Since for every integer $i$ we have $\operatorname{det}\left(U_{i}\right)=1+f^{2}\left(E_{i}\right) \neq$ 0 , thus the inverse exists. It is also easy to see that $\left(U_{i}\right)^{-1}=\operatorname{det}\left(U_{i}\right) U_{i}^{\dagger}$ and $U_{i} U_{i}^{\dagger}=1 / \operatorname{det}\left(U_{i}\right)$. Because of the last equality, the operator (32) can become unitary by a simple rescaling procedure, i.e., $U_{i} \rightarrow \sqrt{\operatorname{det} U_{i}} U_{i}$. Furthermore, according to the Eq. (20) the diagonal form $U_{t}^{d}:=\exp \left(-i H_{d} t\right)$ reads

$$
U_{t}^{d}=\sum_{i=0}^{2^{N}-1} U_{i}^{d}(t) \otimes|i\rangle\langle i|,
$$


where abbreviations

$$
U_{i}^{d}(t)=\operatorname{diag}\left(e^{-i\left(E_{i}^{+}+\alpha f\left(E_{i}\right)\right) t}, e^{-i\left(E_{i}^{-}-\alpha f\left(E_{i}\right)\right) t}\right),
$$

were introduced.

Finally, by combining all the results together we obtain the evolution operator $U_{t}$ of the total system:

$$
U_{t}=\sum_{i=0}^{2^{N}-1} U_{i}(t) \otimes|i\rangle\langle i|
$$

where

$$
\begin{aligned}
U_{i}(t) & =U_{i} U_{i}^{d}(t) U_{i}^{\dagger} \\
& =\frac{1}{1+f_{i}^{2}}\left(\begin{array}{cc}
e_{i}^{+}(t)+e_{i}^{-}(t) f_{i}^{2} & f_{i}\left(e_{i}^{+}(t)-e_{i}^{-}(t)\right) \\
f_{i}\left(e_{i}^{+}(t)-e_{i}^{-}(t)\right) & e_{i}^{+}(t) f_{i}^{2}+e_{i}^{-}(t)
\end{array}\right) .
\end{aligned}
$$

and $e_{i}^{ \pm}(t):=\exp \left(-i\left(E_{i}^{ \pm} \pm \alpha f_{i}\right) t\right)$ with $f_{i} \equiv f\left(E_{i}\right)$. Let us keep in mind that $U_{i} U_{i}^{\dagger}=\mathbb{I}_{Q}$ for each integer $i$.

\section{Reduced dynamics}

We begin with the assumption that $\rho_{Q}$ and $\rho_{E}$ are arbitrary density operators for the open system and its environment, respectively. Furthermore, we consider only factorable initial states $\rho_{Q E}$ of the closed system $Q+E$, i.e., $\rho_{Q E}=\rho_{Q} \otimes \rho_{E}$. Using this assumptions and the explicit form of the evolution operator $U_{t}$ given in the Eq. (37), we have that the density operator $\rho_{Q E}(t)=$ $U_{t} \rho_{Q E} U_{t}^{\dagger}$ at any given time reads

$$
\rho_{Q E}(t)=\sum_{i, j=0}^{2^{N}-1} U_{i}(t) \rho_{Q} U_{j}(t)^{\dagger} \otimes|i\rangle\left\langle i\left|\rho_{E}\right| j\right\rangle\langle j| .
$$

Tracing out the last equation over the environment degrees of freedom, we obtain the reduced dynamics (note $\left.\rho_{Q}(t)=\operatorname{Tr}_{E}\left(\rho_{Q E}(t)\right)\right)$

$$
\rho_{Q}(t)=\sum_{i=0}^{2^{N}-1} \rho_{i} U_{i}(t) \rho_{Q} U_{i}(t)^{\dagger},
$$

where $\rho_{i} \equiv\left\langle i\left|\rho_{E}\right| i\right\rangle$. By introducing matrices $K_{i j}(t)$ such that $K_{i j}(t):=\delta_{i j} \sqrt{\rho_{i}} U_{i}(t)$, the equation above can be written in a more familiar form, that is $\rho_{Q}(t)=$ $\sum_{i j} K_{i j}(t) \rho_{Q} K_{i j}(t)^{\dagger}$. Moreover, it is easily to verify that $\sum_{i j} K_{i j}(t) K_{i j}(t)^{\dagger}=\mathbb{I}_{Q}$. Thus, the Eq. (40) can be thought of as operator sum representation of the state $\rho_{Q}(t)$. The matrices $K_{i j}(t)$ are the Kraus operators. The result (40) is general, i.e., it holds for the arbitrary states $\rho_{Q}$ and $\rho_{E}$. Nevertheless, we made assumption about the form of the initial state $\rho_{Q E}$. This assumption, however, is not essential and does not lead to the limitation of the analysis. The evolution operator (37) was written in a highly manageable form, and by that we mean it can be applied to any given initial state. Of course, if initial correlations are present, then the Eqs. (39), and (40) no longer hold, but the reduced dynamics $\rho_{Q}(t)$ can still easily be obtained. However, we will not focus on this issue in the current paper.

So far, we omitted the explicit dependency on $\beta$ in the introduced quantities. Note, however, that if $U_{i}(t)=U_{i}(t, \beta)$, then $\rho_{Q}(t)=\rho_{Q}(t, \beta)$ and in view of the Eqs. (16), (40), (17) and comments following them, we have

$$
\eta_{t}=\sum_{i, j=0}^{2^{N}-1} M_{i j}(t) \eta_{Q} M_{i j}(t)^{\dagger},
$$

where $\eta_{Q}\left(\eta_{Q}=\rho_{Q}\right)$ is an arbitrary initial state. The Kraus matrices $M_{i j}(t)$ are given by

$$
M_{i j}(t)=\delta_{i j} \sqrt{\rho_{i}} V_{t} U_{i}\left(t, \beta_{e f f}\right) .
$$

The last two equations are the main result of the current paper. It is worth mentioning that the model with a similar properties was studied in [23], where the Authors obtained the operator sum representation, yet the Kraus operator introduced therein involved the time chronological operator.

From the Eqs. (41) and (42) one can learn that the time evolution of the qubit interacting with the rotating magnetic field (2) and in contact with the environment (13) - (14) can be described using two quantum channels, i.e., $\rho_{Q}(t)=T_{t}^{2} \circ T_{t}^{1}\left(\rho_{Q}\right)$. The first channel, that is $T_{t}^{1}$ is given by

$$
T_{t}^{1}:=\sum_{i=0}^{2^{N}-1} M_{i}(t)(\cdot) M_{i}(t)^{\dagger} .
$$

The second one is defined by the simple unitary operation, namely $T_{t}^{2}=V_{t}(\cdot) V_{t}^{\dagger}$. The matrices $M_{i}$ are specified by

$$
M_{i}(t)=\sqrt{\rho_{i}} \exp \left(-i H_{i} t\right),
$$

where the Hamiltonian $H_{i}$ is the generator of the unitary evolution $U_{i}\left(t, \beta_{\text {eff }}\right)$ (see (38)). It is easy to compute that

$$
H_{i}=\left(\begin{array}{cc}
E_{i}-\omega / 2 & \alpha \\
\alpha & -E_{i}+\omega / 2
\end{array}\right),
$$

where $E_{i}$ is the eigenvalues of the operator $V$ and it was defined in the Eq. (27). The channel $T_{t}^{2}$ may by thought of as the convex combination of the unitary channels 
(note $\left.T_{t}\left(\mathbb{I}_{Q}\right)=\mathbb{I}_{Q}\right)$. This type of channels are known as the random unitary channels [24] and they often appears in the study of the pure decoherence.

Surprisingly, the matrix above and therefore the Kraus matrices (44) does not depend on the function $f\left(E_{i}\right)$. However, to compute matrices $\exp \left(-i H_{i} t\right)$ that specified the Kraus matrices (44) we need to diagonalize the Hamiltonians $H_{i}$. The function $f\left(E_{i}\right)$ is hidden in the similarity matrix $U_{i}$ given by the Eq. (33). Observe that the mentioned diagonalization procedure is based on the Riccati equation (matrices $U_{i}$ are composed with the eigenvalues of the solution of the Riccati equation). This approach differs from the standard method based on the characteristic equation. This is a new kind of the diagonalization procedure, so called Riccati diagonalization [25].

\section{SPECIAL CASES}

Now, we look into some common cases that may arise during the examination of the model (12). We will show that in these particular situations the result (41) can be simplified to the well-know expressions.

\section{A. No coupling with the bath}

In the case when the qubit under consideration has no coupling with its environment, i.e., $g_{n}=0$ for all $n \leq N$ we have

a) $E_{i}=\beta_{\text {eff }}$, hence

b) $f_{i} \equiv f=\left(|z|-\beta_{\text {eff }}\right) / \alpha$, where

c) $z:=\alpha+i \beta_{\text {eff }}$.

Moreover, $E_{i}^{ \pm}=\Omega_{i} \pm \beta$, where $\Omega_{i} \equiv \sum_{n} \omega_{n}(-1)^{i_{n}}$. Using this simplification we obtain that $U_{i}\left(t, \beta_{\text {eff }}\right)=$ $e^{-i \Omega_{i} t} \bar{U}(t)$, where

$$
\begin{aligned}
\bar{U}(t) & =\left(\begin{array}{cc}
e^{-i|z| t}+f^{2} e^{i|z| t} & -2 i f \sin (|z| t) \\
-2 i f \sin (|z| t) & f^{2} e^{-i|z| t}+e^{i|z| t}
\end{array}\right) \\
& =I_{2} \cos (|z| t)-i\left(\begin{array}{cc}
\frac{1-f^{2}}{1+f^{2}} & \frac{2 f}{1+f^{2}} \\
\frac{2 f}{1+f^{2}} & -\frac{\left(1-f^{2}\right)}{1+f^{2}}
\end{array}\right) \sin (|z| t) \\
& =I_{2} \cos (|z| t)-i \boldsymbol{\sigma} \cdot \hat{\mathbf{\Omega}} \sin (|z| t) \\
& =\exp (-i|z| \boldsymbol{\sigma} \cdot \hat{\mathbf{\Omega}} t),
\end{aligned}
$$

with $\hat{\boldsymbol{\Omega}}:=\boldsymbol{\Omega} /\|\boldsymbol{\Omega}\|$ and $\boldsymbol{\Omega}:=\left(2 f, 0,1-f^{2}\right)$. As a result, $M_{i j}(t)=\delta_{i j} \sqrt{\rho_{i}} V_{t} \bar{U}(t)$, where we dropped phase factor $e^{-i \Omega_{i} t}$. Therefore $\eta_{t}=U(t) \eta U(t)^{\dagger}$ (as one may anticipated), where

$$
U(t)=\exp \left(-i \omega t \sigma^{z} / 2\right) \exp (-i|z| \boldsymbol{\sigma} \cdot \hat{\boldsymbol{\Omega}} t) .
$$

This is the well-known formula for the (unitary) evolution operator of the system identified by the Hamiltonian $H_{Q}(t, \beta) \equiv H_{Q}(t)$. It is the solution of the differential equation $i \partial_{t} U(t)=H_{Q}(t) U(t)$. Note, that the result (47) corresponds to the Floquet decomposition of the unitary operator generated by the periodic Hamiltonian. Note also that the evolution operator (47) can by written in a more readable form, especially useful for studying the adiabatic approximation (see Sec. (V)), i.e.

$$
U(t)=V_{t} \exp (-i H t),
$$

where the matrix $V_{t}$ is given by the Eq. (17) and $H$ has the form

$$
H=\left(\begin{array}{cc}
\beta_{e f f} & \alpha \\
\alpha & -\beta_{e f f}
\end{array}\right) .
$$

One can easily see that this matrix can be obtained directly from the Eq. (45) if one set $g_{n}=0$ for $n \leq N$.

\section{B. Dephasing}

If the constant $\alpha$ equals zero, then the Hamiltonian $H_{Q E}(t, \beta)$ becomes time-independent. Moreover, in this circumstances $\left[H_{Q}, H_{\text {int }}\right]=0$ hence the open system does not exchange energy with its environment, i.e., the pure decoherence occurs. It poses no problems to check that $U_{i}\left(t, \beta_{\text {eff }}\right)=e^{-i \Omega_{i} t} V_{t}^{\dagger} \exp \left(-i E_{i} \sigma^{z} t\right)$. Therefore, the Kraus operators take the form $M_{i j}(t)=$ $\delta_{i j} \sqrt{\rho_{i}} \exp \left(-i E_{i} \sigma^{z} t\right)$. Just like before the phase factor $e^{-i \Omega_{i} t}$ was drooped. In agreement with (41) the form above of the Kraus matrices leads to the following manifestation of the density matrix $\eta_{t}$

$$
\eta_{11}(t)=\eta_{11}, \quad \eta_{12}(t)=\sum_{i=0}^{2^{N}-1} \rho_{i} e^{-i 2 E_{i} t} \eta_{12}
$$

Naturally, $\eta_{22}(t)=1-\eta_{11}(t)$ and $\eta_{21}(t)=\eta_{21}(t)^{*}$. Note that the coherence $C(t):=\left|\eta_{12}(t)\right|$ does not decay exponentially (or anyhow for finite $N$ ) in the long time regime, but manifests oscillating behavior.

From the considerations above one can easily learn that there is a substantial difference between the situation when a transfer of the energy from the qubit to its bath is not present and the case when the qubit is not coupled to the environment. Let us also observe that in the model we study it is not possible to construct a situation when the energy exchange in not present $(\alpha=0)$ and yet the magnetic field is rotating. This follows from the fact that if $\alpha=0$ then the Hamiltonian $H_{Q E}(t, \beta)$ does not depend on $\omega$. 


\section{Low temperature regime}

From the mathematical point of view the zero (low) temperature limit is the most subtle special case. This comes from the fact that most results obtained by using the known approximation methods in the open quantum systems theory, e.g., the Markovian or the singular coupling limit, cannot by extrapolated to this regime. Luckily, the exactly solvable models are unhampered by this difficulties.

To derive the exact reduced dynamics in the low temperature domain we begin with rewriting the density operator $\rho_{E}$ in a more suitable form in contrary with the one given by Eq. (31). According to the result obtained in [16] one may write

$$
\rho_{E}=\bigotimes_{n=1}^{N} \frac{1}{2}\left(\mathbb{I}_{Q}+\beta_{n} \sigma^{z}\right),
$$

where $\beta_{n}=\tanh \left(-\omega_{n} / k T\right)$. From equality (51) one may readily see when $T \rightarrow 0$, then

$$
\rho_{i}=\frac{1}{2^{N}} \prod_{n=1}^{N}\left(1+\beta_{n}(-1)^{i_{n}}\right) \rightarrow \delta_{i 0},
$$

since $\beta_{n} \rightarrow 1$ as $T \rightarrow 0$. All this means that in the low temperature domain the heat bath is its ground state, i.e., $\rho_{E}=\left|0_{E}\right\rangle\left\langle 0_{E}\right|$. As an immediate result we obtain the following form of the reduced dynamics (41)

$$
\lim _{T \rightarrow 0} \eta_{t}=W_{t} \eta W_{t}^{\dagger},
$$

where $W_{t}:=V_{t} U_{0}\left(t, \beta_{e f f}\right)$. Interestingly, the evolution of the open system in question is unitary, which is rather unusual. This is a direct consequence of the fact that for every integer $i$ the operator $U_{i}(t)$ specified by the Eq. (38) is unitary. This means that in the low temperature regime the dissipative properties of the heat bath (13) - (14) are frozen.

\section{Equal Couplings and Frequencies}

Henceforward and without essential loss of generality we assume that $\omega_{n}=\Omega$ and $g_{n}=g$ for $n \leq N$, i.e., couplings constants and the frequencies of the spins of the bath are equal. In this situation $E_{i}=g(N-2 k)+\beta$, where $k$ is the Hamming weight of the integer number $i$ (i.e., a number of nonzero element in a binary expansion of $i)$. Since there are $\left(\begin{array}{l}N \\ k\end{array}\right)$ integer number $i \in\left[0,2^{N}-1\right]$ with the same Hamming weight $k$, the channel (43) takes the form

$$
T_{t}^{1}=\sum_{k=0}^{N}\left(\begin{array}{l}
N \\
k
\end{array}\right) M_{k}(t)(\cdot) M_{k}(t)^{\dagger},
$$

with $M_{k}(t)$ given by Eq. (44). Note, in this case $\rho_{k}$ (see Eq. (51)) simplified to the form

$$
\rho_{k}=\frac{1}{2^{N}}(1+\delta)^{N-k}(1-\delta)^{k},
$$

where $\delta:=\tanh (-\Omega / k T)$.

\section{APPLICATION: ADIABATIC APPROXIMATION}

The adiabatic theorem $[26,27]$ for the closed quantum system specified by the Hamiltonian $H(t)$ in its basics variant (i.e., discrete and no degenerate spectrum $\sigma(H(t)))$ states that if $H(t)$ varies slowly (for rigorous meaning of that see e.g., [28] and references therein) and if the system is initially prepared in one of the eigenstates of $H(0)$, say $\left|\psi_{n}(0)\right\rangle$, then at any given time $t$ the probability $\left|\left\langle\psi_{n}(t) \mid \psi(t)\right\rangle\right|^{2}$ of finding it in the eigenstate $\left|\psi_{n}(t)\right\rangle$ (an eigenvector of $H(t)$ ) is equal to 1 . Therefore, one may easily introduce the quantity $F(t)$ that measures the validity of the adiabatic approximation, namely

$$
F(t)=\operatorname{Tr}\left(\rho(t) \rho_{\psi}(t)\right),
$$

where $\rho_{\psi}(t)=\left|\psi_{t}\right\rangle\left\langle\psi_{t}\right|$ and $\rho(t)=U_{t} \rho(0) U_{t}^{\dagger}$ with $\rho(0)=$ $\left|\psi_{0}\right\rangle\left\langle\psi_{0}\right|$. Here, $\left|\psi_{t}\right\rangle$ is the eigenvector of $H(t)$ for $t \geq 0$ and $U_{t}$ represents the (unitary) evolution operator for the closed system. If the system is evolving adiabatically then $F(t)=1$. Note that definition of the quantity $F(t)$ coincides with the standard definition of the quantum fidelity $F\left(\rho(t), \rho_{\psi}(t)\right)$ between pure states $\rho(t)$ and $\rho_{\psi}(t)$ [29]. Since this is a two dimensional case it also coincides with the definition of the super fidelity [30, 31].

Observe that the Eq. (56) is very useful since it can be easily applied both to the closed and to the open quantum systems. In the last case we need to replace the equality $\rho(t)=U_{t} \rho(0) U_{t}^{\dagger}$ representing the evolution of the closed system by the channel describing evolution in time of the open system. More precisely, $\rho(t)=T_{t}(\rho(0))$, where $T_{t}$ is TP-CP quantum operation. In the current section we use the Eq. (56) in order to investigate behavior of the model (12) in the adiabatic regime. To make the paper self-contained we briefly discuss the adiabatic approximation for the system (1) first.

\section{A. The closed spin system case}

For the sake of simplicity we put $\beta=\beta_{0} \cos \varphi$ and $\alpha=\beta_{0} \sin \varphi$ for certain $\varphi$ and $\beta_{0}$. This assumptions lead to the following form of the Hamiltonian (3)

$$
H_{Q}(t)=\beta_{0}\left(\begin{array}{cc}
\cos \varphi & e^{-i \omega t} \sin \varphi \\
e^{i \omega t} \sin \varphi & -\cos \varphi
\end{array}\right)
$$


It can be easily verified that the matrix above has the eigenvalues $E_{ \pm}= \pm \beta_{0}$. The corresponding eigenvectors $\left|\psi_{t}^{ \pm}\right\rangle$are given by

$$
\left|\psi_{t}^{+}\right\rangle=\left(\begin{array}{c}
\cos (\varphi / 2) \\
e^{i \omega t} \sin (\varphi / 2)
\end{array}\right), \quad\left|\psi_{t}^{-}\right\rangle=\left(\begin{array}{c}
\sin (\varphi / 2) \\
-e^{i \omega t} \cos (\varphi / 2)
\end{array}\right)
$$

The density matrix $\rho_{+}(t)=\left|\psi_{t}^{+}\right\rangle\left\langle\psi_{t}^{+}\right|$takes the form

$$
\rho_{+}(t)=\left(\begin{array}{ll}
\cos ^{2}(\varphi / 2) & \frac{1}{2} e^{-i \omega t} \sin \varphi \\
\frac{1}{2} e^{i \omega t} \sin \varphi & \sin ^{2}(\varphi / 2)
\end{array}\right)
$$

Observe that $\rho_{+}(t)=V_{t} \rho_{+}(0) V_{t}^{\dagger}$, where $V_{t}$ is specified in the Eq. (17). According to the Eq. (48) the density matrix $\rho(t)$ at any given time $t$ reads $\rho(t)=$ $V_{t} \bar{U}(t) \rho_{+}(0)\left(V_{t} \bar{U}(t)\right)^{\dagger}$, thus the fidelity (56) takes the form

$$
\begin{aligned}
F(t) & =\operatorname{Tr}\left(\bar{U}(t) \rho_{+}(0) \bar{U}(t)^{\dagger} \rho_{+}(0)\right) \\
& =\left\|\bar{U}(t) \rho_{+}(0)\right\|_{F}^{2},
\end{aligned}
$$

where $\|\cdot\|_{F}$ is the Frobenius (Euclidean) norm, i.e., $\|A\|_{F}^{2}:=\operatorname{Tr}\left(A A^{\dagger}\right)$.

It is well-known that for the system governed by the Hamiltonian (57) condition that guarantees the adiabatic evolution is $\beta_{0} \gg \omega$. This statement is very intuitive, it means that the magnetic field rotates slowly, in comparison with the phase of the state vector (for more detail discussion see e.g., [32]). We can also see this from the Eq. (60). Indeed, if one introduces the adiabatic parameter $x:=\omega / 2 \beta_{0}$, then in agreement with (48) and (60) we have

$$
F(t)=1-\frac{x^{2}}{1+x^{2}} \sin ^{2}(\Omega(x) t),
$$

where $\Omega(x):=\beta_{0} \sqrt{1+x^{2}}$. Without loss of generality we set $\varphi=\pi / 2$ in the equation above. The parameter $x$ measures how slowly the magnetic field rotates in $\beta_{0}$ units, thus in the adiabatic limits (i.e., $x \rightarrow 0$ ) the second term in the Eq. (61) can be neglected and therefore $F(t) \simeq 1$.

\section{B. The open system case}

By analogy to the closed spin case discussed above one may write the fidelity (56) for the open system (12) as

$$
\begin{aligned}
F(t) & =\operatorname{Tr}\left(T_{t}^{2} \circ T_{t}^{1}\left(\rho_{+}(0)\right) \rho_{\psi}(t)\right) \\
& =\sum_{k=0}^{N}\left(\begin{array}{l}
N \\
k
\end{array}\right) \rho_{k} F_{k}(t),
\end{aligned}
$$

where $F_{k}(t):=\left\|U_{k}(t) \rho_{+}(0)\right\|_{F}^{2}$. The channels $T_{t}^{1}$ and $T_{t}^{2}$ are specified in the Eq. (43) and comments bellow, respectively. The unitary matrix $U_{k}$ takes the form $U_{k}=$ $\exp \left(-i H_{k} t\right)$, with $H_{k}$ given by the Eq. (45). If we put $\varphi=\pi / 2$ as before, then

$$
F_{k}(t)=1-\frac{x_{k}^{2}}{1+x_{k}^{2}} \sin ^{2}\left(\Omega\left(x_{k}\right) t\right),
$$

where $x_{k}:=G(N-2 k) / \beta_{0}-x$. Using this and Eq. (62) we obtain that $F(t)=1-R(t)$, where $R(t)$ reads

$$
R(t)=\sum_{k=0}^{N}\left(\begin{array}{l}
N \\
k
\end{array}\right) \frac{x_{k}^{2}}{1+x_{k}^{2}} \rho_{k} \sin ^{2}\left(\Omega\left(x_{k}\right) t\right) .
$$

From this equation one can readily see that in the adiabatic domain $(x \rightarrow 0)$ we have $R(t) \neq 0$ and thus $F(t)<1$. Therefore, the standard condition that leads to the adiabatic behavior of the closed system (1) does not guarantee that in the case of the open system model (12) it holds true as well. However, if one additionally assumes that coupling with the environment is weak, in comparison with the energy split between the states $|0\rangle$ and $|1\rangle$ of the qubit in question, that is to say if $G / \beta_{0} \ll 1$, then $x_{k} \ll 1$ (for finite $N$ ) and $R(t) \simeq 0$, thus $F(t) \simeq 1$.

\section{SUMMARY}

In this paper we investigated a qubit in contact with the spin environment and interacting with a rotation magnetic field. The considered model was constructed under a set of assumptions which allow for its exact treatment. We hope that despite the mathematical character the results of the paper may serve as starting point for further investigations. The exact models can not only provide reasonable approximate description of real systems, as it is the case for the pure dephasing, but are often used as a basis and inspiration for various improvements [33]. Although the paper is mainly focused on mathematical aspects it also includes the example of a natural application of the model in question. This example relates our model to important problems of physics such as e.g. the problem of the adiabatic approximation for the open quantum systems [34] or the adiabatic quantum computation [35].

We provided the exact reduced dynamics for the system mentioned above. In contrast to the standard methods available in the theory of the open quantum systems our approach was based on the block operator matrices theory. In particular, we resolved the algebraic Riccati equation associated with the Hamiltonian defining the model under consideration. We wish to stress out that the method we used in the current paper, although applied to the particular model, is general. Nevertheless, its usefulness relies on the ability of solving the Riccati equation. At the present time it is a very difficult task, even for the simple systems. 
At this point one can ask: how relevant is it to assume a spin environment, instead of a bosonic one, to solve the problem exactly? Is it possible to do so for bosonic bath? One may pose a more general question. What is a connection between the possibility of obtaining the exact reduced dynamics of the qubit in question and the Hamiltonian specifying the bath? This problem was already addressed in [8]. The results of this paper as well as the analysis we carried out suggest that this problem is at least as difficult as resolving the Riccati equation associated with the total Hamiltonian.

Furthermore, we studied the adiabatic approximation for the model in question. It was shown that the standard condition that guarantees the adiabatic evolution in the the case of the closed systems is not valid for the open system generalization. This is not an unexpected result. It is interesting, however, that the aforementioned condition does ensures the adiabatic behavior of the open system under consideration in the weak coupling limit.

\section{ACKNOWLEDGMENTS}

The author would like to thank the anonymous referees for their comments. The author also thank Jerzy Dajka and Jarosław Adam Miszczak for helpful suggestions. This work was supported by the Polish Ministry of Science and Higher Education under the grant number N N519 442339.

\section{Appendix A: Proof of the Eq. (16)}

In order to prove the equation (16) let us note that the Hamiltonian (12) satisfies the following condition

$$
H(t, \beta)=e^{i K t} H(\beta) e^{-i K t},
$$

where $K=-\frac{\omega}{2} \sigma_{3} \otimes \mathbb{I}_{E}$. This can be easily proven using the Baker-Campbell-Hausdorff formula [36]. As was shown, in [2] every quantum system with Hamiltonian $H(t, \beta)$ satisfying (A1) for some Hermitian operator $K$ evolves

$$
U_{t}(\beta)=e^{i K t} e^{-i H_{e f f}(\beta) t}, \quad H_{e f f}(\beta):=H(\beta)+K .
$$

Note that in general $[H(\beta), K] \neq 0$ and therefore $\left[H_{e f f}(\beta), K\right] \neq 0$. In our case, from equation (12) we learn that $H(\beta)=\left(\beta \sigma_{3}+\alpha \sigma_{1}\right) \otimes \mathbb{I}_{E}$, thus

$$
\begin{aligned}
H_{e f f}(\beta) & =\left(\beta \sigma_{3}+\alpha \sigma_{1}\right) \otimes \mathbb{I}_{E}-\frac{\omega}{2} \sigma_{3} \otimes \mathbb{I}_{E} \\
& =\left(\left(\beta-\frac{\omega}{2}\right) \sigma_{3}+\alpha \sigma_{1}\right) \otimes \mathbb{I}_{E} \\
& =H\left(\beta-\frac{\omega}{2}\right) .
\end{aligned}
$$

From equations (A2) and (A3) we have

$$
U_{t}(\beta)=e^{i K t} U_{t}\left(\beta-\frac{\omega}{2}\right),
$$

where $U_{t}(\beta)$ is the evolution operator generated by $H(t, \beta)$. Let $\hat{\rho}_{t}(\beta)$ and $\hat{\eta}_{t}$ be a density operator for the closed system $Q+E$ associated with the Hamiltonian $H(\beta)$ and $H(t, \beta)$ respectively in some arbitrary time $t$. Let us also assume that $\hat{\rho}_{0}(\beta)=\hat{\eta}_{0} \equiv \hat{\rho}$. Using equation (A4) one can easily see that

$$
\begin{aligned}
\hat{\eta}_{t} & =U_{t}(\beta) \hat{\rho} U_{t}^{\dagger}(\beta) \\
& =e^{i K t} U_{t}\left(\beta-\frac{\omega}{2}\right) \hat{\rho} U_{t}^{\dagger}\left(\beta-\frac{\omega}{2}\right) e^{-i K t} \\
& =\hat{V}_{t} \hat{\rho}_{t}\left(\beta-\frac{\omega}{2}\right) \hat{V}_{t}^{\dagger},
\end{aligned}
$$

where we introduced $\hat{V}_{t}=e^{i K t}$. To end the proof we will show that if $\hat{A}_{1}, \hat{A}_{2} \in B(\mathcal{H} \oplus \mathcal{H})$ are a $2 \times 2$ block operator matrix of the form $\hat{A}_{i}=A_{i} \otimes \mathbb{I}_{E},(i=1,2)$ and $\hat{B}=\left[\hat{B}_{i j}\right] \in B(\mathcal{H} \oplus \mathcal{H})$ then

$$
\operatorname{Tr}_{E}\left(\hat{A}_{1} \hat{B} \hat{A}_{2}\right)=A_{1} \operatorname{Tr}_{E}(\hat{B}) A_{2} .
$$

Equation (A6) follows from the linearity of the trace $\operatorname{Tr}(\cdot)$ operation and the definition (6) of partial trace. Note that $\hat{V}_{t}=V_{t} \otimes \mathbb{I}_{E}$, where $V_{t}$ is given by the equation (17), thus taking partial trace of the equation (A5) and using (A6) we obtain (16) with $V_{t}$ given by (17).
[1] A. Galindo and P. Pascual, Quantum Mechanics vol 2 (Springer-Verlag, Berlin, Germany, 1990).

[2] B. Traller, Advanced visual quantum mechanics (Springer Science+Business Media, Inc., 2005).

[3] H. P. Breuer and F. Petruccione, The Theory of Open Quantum Systems (Oxford University Press, USA, 2002).

[4] A. J. Leggett, et al., Rev. Mod. Phys. 59, 1 (1987).

[5] W. H. Zurek, Rev. Mod. Phys. 75, 715 (2003).

[6] I. Bengtsson and K. Życzkowski, Geometry of Quantum States: An Introduction to Quantum Entanglement (Cambridge University Press, Cambridge, U.K., 2006).
[7] B. Gardas, Almost pure decoherence, Master's thesis, Institute of Physics University of Silesia (2009).

[8] B. Gardas, J. Math. Phys. 51, 062103 (2010).

[9] J. Dajka, M. Mierzejewski, and J. Łuczka, Phys. Rev. A 79, 012104 (2009).

[10] J. Dajka, M. Mierzejewski, and J. Łuczka, Phys. Rev. A 77, 042316 (2008).

[11] R. Alicki and K. Lendi, Quantum dynamical semigroups and applications, Lecture notes in physics (Springer, Berlin, Germany, 1987).

[12] P. Štelmachovič and V. Bužek, Phys. Rev. A 64, 062106 (2001); Phys. Rev. A 67 
(2003).

[13] H. Hayashi, G. Kimura, and Y. Ota, Phys. Rev. A 67, 062109 (2003).

[14] H. Langer and C. Tretter, J. Operator Theory 39, 339 (1998).

[15] V. Adamjan, H. Langer, and C. Tretter, Journal of Functional Analysis 179, 448 (2001).

[16] H. Krovi, O. Oreshkov, M. Ryazanov, and D. A. Lidar, Phys. Rev. A 76, 052117 (2007).

[17] N. Arshed, A. H. Toor, and D. A. Lidar, Phys. Rev. A 81, 062353 (2010).

[18] C. Kittel, Introduction to Solid States Physics (John Wiley \& Sons, Inc. NY, 1996).

[19] G. A. Álvarez and D. Suter, Phys. Rev. Lett. 104, 230403 (2010).

[20] T. D. Ladd, F. Jelezko, et al., Nature 464, 45 (2010).

[21] A. I. Lvovsky, B. C. Sanders, and W. Tittel, Nature Photonics 3, 706 (2009).

[22] J. H. Reina, L. Quiroga, and N. F. Johnson, Phys. Rev. A 65, 032326 (2002); P. Neumann, et al., Nature Physics 6, 249 (2010).

[23] M. Cetinbas and J. Wilkie, Physics Letters A 372, 1194 (2008).
[24] B. Rosgen, J. Math. Phys. 49, 102 (2008).

[25] K. Fujii and H. Oike, Int. J. Geom. Meth. Mod. Phys. 7 (2010), accepted, arXiv:1004.1207v2.

[26] K.-P. Marzlin and B. C. Sanders, Phys. Rev. Lett. 93, 160408 (2004).

[27] D. M. Tong, et al., Phys. Rev. Lett. 95, 110407 (2005).

[28] J. Avron and A. Elgart, Commun. Math. Phys. 203, 445 (1999).

[29] R. Jozsa, J. Mod. Opt. 41, 2315 (1994).

[30] Z. Puchała and J. Miszczak, Phys. Rev. A 79, 024302 (2009).

[31] Z. Puchała, et al., Quantum Inf. Comput. 9, 0103 (2009).

[32] D. Griffiths, Introduction to quantum mechanics (Prentice Hall, Inc., 1995).

[33] D. I. Schuster, et al., Nature 445, 515 (2007).

[34] M. S. Sarandy and D. A. Lidar, Phys. Rev. A 71, 012331 (2005).

[35] D. A. Lidar, Phys. Rev. Lett. 100, 160506 (2008).

[36] A. Galindo and P. Pascual, Quantum Mechanics vol 1 (Springer-Verlag, Berlin, Germany, 1990). 